\title{
Reduced risk-taking behavior during frontal oscillatory theta band
}

\section{neurostimulation}

\author{
Aline M. Dantas 1,2,3*, Alexander T. Sack 1,2,4, Elisabeth Bruggen ${ }^{3}$, Peiran Jiao 5, Teresa \\ Schuhmann 1,2
}

1. Section Brain Stimulation and Cognition, Department of Cognitive Neuroscience, Faculty of Psychology and Neuroscience, Maastricht University, Maastricht, The Netherlands.

2. Maastricht Brain Imaging Centre, Maastricht University, Maastricht, The Netherlands.

3. Department of Marketing and Supply Chain Management, School of Business and Economics, Maastricht University, Maastricht, The Netherlands.

4. Department of Psychiatry and Neuropsychology, School for Mental Health and Neuroscience (MHeNs), Brain + Nerve Centre, Maastricht University Medical Centre+ (MUMC+), Maastricht, The Netherlands.

5. Department of Finance, School of Business and Economics, Maastricht University, Maastricht, The Netherlands.

*Corresponding author

E-mail: a.dantas@maastrichtuniversity.nl 


\begin{abstract}
Background: Most of our decisions involve a certain degree of risk regarding the outcomes of our choices. People vary in the way they make decisions, resulting in different levels of risk-taking behavior. These differences have been linked to prefrontal theta band activity. However, a direct functional relationship between prefrontal theta band activity and risk-taking has not yet been demonstrated.
\end{abstract}

Objective: We used noninvasive brain stimulation to test the functional relevance of prefrontal oscillatory theta activity for the regulatory control of risk-taking behavior.

Methods: In a within-subject experiment, 32 healthy participants received theta [4-8 Hertz $(\mathrm{Hz})$ ], gamma $(30-50 \mathrm{~Hz})$, and sham transcranial alternating current stimulation (tACS) over the left prefrontal cortex (lPFC). During stimulation, participants completed a task assessing their risktaking behavior as well as response times and sensitivity to value and outcome probabilities. Electroencephalography (EEG) was recorded before and immediately after stimulation.

Results: Theta-band, but not gamma-band or sham, tACS led to a significant reduction in risk-taking behavior, indicating a frequency-specific effect of prefrontal brain stimulation on the modulation of risk-taking behavior. Moreover, theta-band stimulation led to increased response times and decreased sensitivity to reward values. EEG data analyses did not show an increase in power in the stimulated frequencies.

Conclusion: These findings provide direct empirical evidence for the effects of prefrontal theta-band stimulation on behavioral risk-taking regulation.

Keywords: tACS, risk-taking behavior, theta activity, theta frequency, decision neuroscience 


\section{Introduction}

3 and outcome probabilities. Take for example financial investments, driving above the speed limit

4 or simply trying a new cuisine. In all of these situations, and many others, the outcomes of our

5 decisions and actions cannot be predicted with absolute certainty. The decision-maker exhibits

6 risk-taking behavior in these situations. Risk-taking is inevitable and may not only have

7 (un)desired personal, but also social and economic impacts (1). Therefore, the regulatory control

8 of risk-taking behavior is of utmost importance for human decision-making.

9 During decision-making under risk, a complex mechanism is at work. This mechanism

10 codes and flexibly evaluates the context, outcome probabilities and previous information to define

11 the optimal level of risk to be taken (2). Despite what is expected from pure rational models, risk-

12 taking behavior is not consistent across different contexts and the most optimal decision is often

13 rejected (3). These inconsistencies are likely a consequence of the complexity of the neural

14 mechanisms involved in risk-taking behavior and the control thereof, which have been extensively

15 explored by previous studies (e.g. 3-6). Namely, risk-taking behavior is the result of a complex

16 interplay between emotional responses to possibilities of reward (limbic activity) and inhibition of

17 such responses via activation of frontal control regions (7). Among these, the ventromedial

18 prefrontal cortex (VMPFC) and dorsolateral prefrontal cortex (DLPFC) are critical areas,

19 responsible for signaling the need of strategy adjustment and executive control, respectively (8).

20 However, the exact mechanism underlying such signaling processes remains unclear.

21 Electroencephalography (EEG) studies have shown that participants with a higher theta

22 power [4-8 Hertz $(\mathrm{Hz})]$ in the right prefrontal cortex (rPFC) compared to the $1 \mathrm{PFC}$, i.e. a higher

23 frontal theta-band asymmetry, displayed more risk-taking behavior during gambling tasks $(9,10)$. 
24 These results are consistent with studies showing a positive correlation between error detection,

25 cognitive control and increased right VMPFC theta power (11). Moreover, theta oscillations are

26 involved in neural network communication when cognitive control is required (12). Prefrontal

27 theta oscillations may therefore represent part of the signaling mechanism by which the VPMFC

28 recruits the DLPFC, in case recruitment of regulatory mechanisms is needed because a risky

29 context is detected. However, although these EEG studies indicate that theta oscillations are related

30 to risk taking behavior, the functional behavioral relevance of this oscillatory pattern in the

31 regulation of risk-taking has yet not been shown.

32 Noninvasive brain stimulation, such as transcranial alternating current stimulation

33 (tACS), offers the possibility of inducing temporary oscillatory patterns in specific brain regions,

34 by applying changing electric currents on the scalp transiently modulating brain activity. This

35 allows to probe the relationship between frequency patterns and behavioral responses (13). To

36 investigate the role of theta band frontal asymmetry in risk-taking behavior, Sela and colleagues

37 (2012) applied theta-band tACS over right and left DLPFC while participants performed the

38 Balloon Analog Risk Task. After left, but not right, DLPFC theta-band stimulation an increase in

39 risk-taking behavior was found. This was not in line with prior EEG studies hypothesizing that

40 right DLPFC stimulation increases risk-taking behavior, by increasing frontal asymmetry, while

41 left DLPFC tACS reduces risk-taking behavior due to an increase in theta-band activity in the left

42 hemisphere and consequent asymmetry reduction $(6,9)$. Sela and colleagues $(2010)$ speculated that

43 their findings may be due to a disruption of interhemispheric balance in participant's natural frontal

44 asymmetry (14). The authors were not able to make conclusions about the frequency specificity of

45 the stimulation, as no control frequencies were applied(15). Moreover, they opted for using the

46 Balloon Analog Task to estimate risk. This task mostly measures impulsivity and evaluates 
47 uncertainty, rather than risk, which is a different economic construct (16), since the probabilities

48 are not explicit to participants.

The present study aims at investigating the functional relationship between frontal theta-

50 band oscillations and risk-taking behavior. Although previous studies $(9,10)$ have shown a

51 correlation between resting state frontal theta band asymmetry and risk-taking behavior, no direct

52 causal relationship has thus far been shown. We therefore applied tACS to the left DLPFC in theta-

53 band $(6.5 \mathrm{~Hz})$, and gamma-band $(40 \mathrm{~Hz})$ frequency as well as sham stimulation while participants

54 performed a risk-taking task. We chose gamma-band tACS as a control frequency since it has not

55 been linked to risk-taking behavior thus far. We also implemented a new behaviorally-controlled

56 risk-taking protocol paired with financial incentives for more robust measures of risk-taking

57 behavior.

To monitor possible power changes in the stimulated frequencies and to investigate their

59 functional relationship with the behavioral results, we implemented EEG measurements before

60 and immediately after the transcranial brain stimulation. We hypothesized that, compared to sham

61 and gamma-band stimulation, theta-band stimulation to the left DLPFC decreases risk-taking

62 behavior, confirming the central regulatory role of theta-frequencies on the electrophysiological

63 mechanism underlying the modulation of risk-taking behavior $(9,17)$.

64

65 Material and methods

66 Participants

Thirty-two healthy, right-handed students (16 female, mean age 23.8 years, range 18-31

68 years, $\mathrm{SD}=3.45$ ), participated in this study. All participants had normal or corrected-to-normal

69 vision and gave written informed consent after being introduced to the experiment. They were 
screened for tACS safety, following the recommended procedures of Antal and colleagues (2017)

71 (18), screening for e.g. skin diseases, implants, neurological disorders, pregnancy and medication.

The study was approved by the Ethics Review Committee Psychology and Neuroscience (ERCPN) of Maastricht University, The Netherlands (ERCPN 188_07_02_2018). Participants were compensated, in the form of vouchers with monetary value, based on the choices they made and luck in the risk-taking task, and for participating in the experiment. The stimulation was well tolerated by 31 out of 32 participants. One participant reported skin redness in the area of the stimulation after participating in session 1 and therefore decided to stop participation in the experiment. The results of this participant were excluded from the analyses.

\section{Procedure} explained in the following sections. The order of stimulation conditions (interventions) was

84 randomized across participants.

89 information, signed the safety pre-experimental check and the written consent and. In each session,

90 participants were instructed about the experimental procedures and task and positioned at the

91 workstation where the tACS and EEG electrodes were placed. EEG was measured before and after

92 the stimulation. During tACS, participants had to perform a computerized gambling task named

93 Maastricht Gambling Task. 
Participants were informed that at the end of the session, one random trial of the task,

95 which is composed by multiple trials (more details will follow in the corresponding session) would

96 be selected for payment. They were asked to use an online random number generator to select the

97 number of the trial that would be paid out. This was done in each of the three sessions. During the

98 task, experimental currency was expressed as points. Every point earned in the selected trial was

99 converted to $€ 0.10$. All participants received also a participation fee of $€ 7.5$ per hour (1.5 hour

100 per session). The payments varied between $€ 33.75$ and $€ 63.75$ and were made only after the third

101 session. All task details and payment rules were explained before the task (Fig1).

102

\section{Maastricht Gambling Task}

A customized experimental protocol to elicit and access risk-taking behavior was

105 developed based on the widely used "Risk task" (19) also known as Cambridge Gambling Task

106 (CGT). The CGT is a valid measurement of risk-taking behavior $(20,21)$, controls for impulsivity

107 and was used in multiple studies using noninvasive brain stimulation (4,22-24). However, the

108 CGT does not control for memory and wealth effects because the trials are not independent,

109 meaning that participants carry gains and losses from the previous trials; and it is confounded by

110 loss aversion, as participants could lose points during the task.

111 Therefore, we developed a revised protocol named the Maastricht Gambling Task (MGT).

112 This computerized task presents six boxes (see Fig 2 for an example screen) to the participant,

113 which can be either colored pink or blue. The number of pink boxes is randomized and ranges

114 from 1 to 5 , with the remaining boxes being blue. One of the colored boxes hides a token

115 (represented by a yellow X) and the participant has to guess the color of the box that hides the

116 token by choosing left (pink) or right (blue). 
118 is correct (hit). A wrong guess results in zero payoff. For example, in Fig 2, the trial offers a chance

119 of $3 / 6(50 \%)$ of earning 50 points if pink is chosen, and $3 / 6(50 \%)$ of earning 100 points if blue is

120 chosen. The bet values were selected randomly among five different values $(5,25,50,75$ or 100$)$

121 for each color in each trial independently. The participant's goal was to obtain the maximum of

122 points in each trial. To remove the impact of loss aversion, the MGT does not allow for losses.

123 Trials have no inter-dependency in the MGT. Payoffs are calculated for each trial independently,

124 and are not cumulated over trials. This avoids memory and wealth effects.

125 Finally, participants see all the possible combinations of the five different bet values with

126 the different probabilities, resulting in 125 unique trials, therefore participants can perceive that

127 there is no deception and all possibilities are randomly assigned. Each trial is displayed twice,

128 which yields a total of 250 trials in random order to guarantee consistent results. Participants had

129 an average of consistency of $100 \%$ in the probabilities chosen and $93 \%$ (standard deviation $4 \%$,

130 median 93\%) in terms of risk-taking and average value chosen across the repeated trials. No

131 participant made significantly inconsistent decisions comparing the two repetitions of each trial in

132 a session.

133 The token's location, color distribution and bet values are determined independently and

134 randomly across trials. With this, we guaranteed that there was no deception and full

135 randomization. This also minimizes the chance of any specific strategy development. All

136 participants were informed explicitly that there was no winning strategy since all results were

137 random. 
Example screen presenting a trial where choosing blue offers a payout of 100 points with a probability of $3 / 6(E V=50)$ and pink offers a payout of 50 points and a probability of $3 / 6(E V=25)$. In this example the participant chose the highlighted option (blue) and the token (yellow $x$ ) is revealed to be hidden behind one of the blue boxes. In this example the participant gained 100 points as it is presented in white.

When a participant chooses a color, the choice is highlighted and the position of the token

147 is revealed (Fig 2). Therefore, in this same example, as the participant chose blue and the token

148 was hidden behind a blue box, the participant gets 100 points (as indicated in the white text on the 149 right).

To gain more insight on the different types of trials, we divided them into three clusters

151 according to the differences (or contrasts) in expected values offered by the two options (Pink and

152 Blue), which could capture the difficulty of making a choice in the trial. The lower this difference

153 the more difficult it is for a subject to make a choice. This led to the division of trials in these

154 clusters: low, medium and high contrast. In our analysis, we excluded trials with no difference in

155 expected value, since this group of options includes less trials than the remaining clusters and

156 would not allow balanced analyses. Trials with one strictly dominant option, meaning (for

157 simplicity) trials where the options have differences in expected value $>|65|$ were excluded. This

158 exclusion was made since these were considered non-informative, because these choices are

159 considered obvious and would hardly be affected by any environmental or intrinsic factor. In total

160204 out of 250 trials were analyzed per session. The cluster division can be seen in detail in the S1

161 Table.

\section{Transcranial alternating current stimulation}

We aimed at stimulating the left DLPFC. A small circular (diameter: $2.1 \mathrm{~cm}$, thickness:

$1652 \mathrm{~mm}$ ) and a large (Outer diameter: $11 \mathrm{~cm}$; Inner diameter: $9 \mathrm{~cm}$, thickness: $2 \mathrm{~mm}$ ) rubber ring tACS

166 electrode (neuroConn, Ilmenau, Germany) were placed using conductive gel (Ten20 conductive 
167 Neurodiagnostic electrode paste, WEAVER and company, Aurora CO, USA) onto the left DLPFC,

168 with the small electrode positioned over F3 (based on the international 10-20 EEG system) and

169 the large electrode around it. Electrode positioning and tACS simulation were modelled with

170 SimNIBS (25), as shown in Fig 3.

Fig 3 - SimNIBS tACS simulation

Left lateral (A), frontal (B) view and of the stimulation and coronal cut at F3 to show the potential subcortical reach of the stimulation $(C)$. Colors stand for the normalized electric field [0-0.35], meaning that the red areas are the areas where the electric stimulation has a higher incidence.

This ring electrode montage enables a higher spatial focality as compared to standard which we created stimulus protocols on Matlab2018b (The Mathworks Inc., Massachusetts, USA)

181 for each condition. Stimulation frequency and intensity were set to $6,5 \mathrm{~Hz}$ (theta-range stimulation)

182 and $40 \mathrm{~Hz}$ (gamma-range stimulation) and a stimulation intensity of 1,5mA peak to peak, phase

183 offset was set to 0 and 100 cycles were used for ramping up. For sham tACS, the current was

184 ramped up at a $6.5 \mathrm{~Hz}$ frequency for 30 seconds and ramped down immediately after. The

185 impedance of the tACS electrodes was kept below $15 \mathrm{k} \Omega$ during stimulation. The average

186 stimulation time lasted 30 minutes. Participants were blind to the stimulation protocol and the

187 experimental hypotheses. Questionnaires applied after the experimental session confirmed that 188 participants were unaware of the stimulation protocol.

189 Electroencephalography 
192 cortex (P5 and P6), with $\mathrm{Cz}$ being used as reference and the left mastoid used as ground. EEG

193 measurements were done immediately before and after the tACS stimulation, each lasting 3

194 minutes, to measure resting-state theta-band activity (measurement before the stimulation) and the

195 effects of the entrainment (after stimulation). Participants were asked to stay with their eyes closed, 196 relaxed and to avoid any movement.

197 Data was recorded (DC-200 Hz, sampling rate $500 \mathrm{~Hz}$ ) with a BrainAmp Standard EEG 198 amplifier and the BrainVision Recorder software (BrainProducts GmbH, Munich, Germany).

199 Impedance levels were kept below $15 \mathrm{k} \Omega$. Offline preprocessing was conducted using the Fieldtrip 200 toolbox (28) and custom Matlab scripts. EEG recordings were low pass-filtered in the analog 201 domain (cutoff frequency: $250 \mathrm{~Hz}$ ) and then digitized (sampling rate: $1000 \mathrm{~Hz}$ ). Offline 202 preprocessing was performed with a notch-filter $(50 \mathrm{~Hz})$ to remove electrical noise and demean 203 the data over the full dataset. After that, it was segmented into 90 trials of 2 seconds each. Trials 204 with high variance and excessive noise were excluded by visual inspection and variance analyses.

\section{Statistical Methodology}

3) Value and 4) Response time.

\section{Risk}

210 outcomes and the value associated with each outcome. In our experiment, betting on a color $X$ (

$211 X=$ blue or pink) in a trial with probability $p$, and a payoff of $x$ would have an expected payoff of

$212 x p$. For instance, when choosing pink, the probability of being correct (a hit) and getting the reward

213 is equal to the proportion of pink boxes during that trial, and the probability of being incorrect and 
214 getting no reward is equal to the proportion of blue boxes. Therefore, the expected payoff from

215 choosing color $X$ in a trial is given by the following.

216

$$
E(X)=x p
$$

For example, in a trial with 1 blue box with a bet value of 100, and 5 pink boxes with a

218 bet value of 5, the expected payoff for blue and pink are respectively 16.67 and 4.17. This makes

219 blue more attractive for a risk-neutral participant. Therefore, an option is strictly dominant for a 220 risk-neutral participant if it has higher expected payoff. from choosing color $X$ is given by the following.

$$
\operatorname{Var}\left(X_{i}\right)=\sum p(x-E(X))^{2}
$$

230 to as Risk.

$$
\text { Risk }=S D=\sqrt{\operatorname{Var}(X)}
$$

\section{Probability scores}

Previous studies only considered the choice of specific outcome probabilities as an

234 indicator of risk $(4,22,31)$, meaning that in these studies, a choice is typically considered risky if 235 the probability is below $50 \%$ and safe if its probability is above $50 \%$. To allow a more refined 236 analysis of participant's preferences of probabilities, they were transformed into a scale ranging 
with a higher level of uncertainty have positive scores, while safer options have negative scores.

240 These probability scores can be seen in Table 1.

\section{Table 1- Probability scores.}

Higher scores indicate that participants chose the trials with lower probabilities (risk prone), while lower scores indicate that participants chose higher probabilities (risk averse). For example, if a participant choose Blue in a trial where the distribution of Blue boxes is 1/6 (and Pink boxes 5/6) the participant would receive a score 2, indicating that the participant chose the lowest probability possible. If in this same trial the participant chooses Pink, the score would be -2, indicating that this participant chose the highest possible probability.

\begin{tabular}{|c|c|c|c|}
\hline Pink & Blue & Choice & Probability \\
\hline 5 & 1 & Blue & 2 \\
\hline 1 & 5 & Pink & 2 \\
\hline 4 & 2 & Blue & 1 \\
\hline 2 & 4 & Pink & 1 \\
\hline 3 & 3 & Pink & 0 \\
\hline 3 & 3 & Blue & 0 \\
\hline 4 & 2 & Pink & -1 \\
\hline 2 & 4 & Blue & -1 \\
\hline 5 & 1 & Pink & -2 \\
\hline 1 & 5 & Blue & -2 \\
\hline
\end{tabular}

\section{Value and Response Time}

To analyze the average value chosen by a participant in each session, their choices of bet values independent of the trial result (being correct or incorrect) were averaged. That variable is named Value. Furthermore, response times (RT) were also recorded for every decision.

\section{Behavioral data analyses}

The behavioral data were preprocessed using custom Matlab (The Mathworks Inc., Massachusetts, USA). We performed a series of Linear Mixed Model analyses to estimate the 
256 effects of stimulation (sham, theta and gamma) on risk-taking behavior. Our final models were

257 fixed effects models, with participant-specific random effects. All the analyses presented normally

258 distributed residuals, showed no heteroscedasticity, and no observations were removed as outliers.

259 Overall, we constructed linear mixed models where each observation is a unique subject-

260 cell pair. Each cell is a unique combination of session and contrast. That is, 3 sessions by 3 levels

261 clusters (LC (low contrast), MC (medium contrast) and HC (high contrast)), resulting in 9 unique

262 observations (cells) per subject. The resulting models can be represented as the following:

263

$$
Y_{i j}=\left(\gamma_{0}+u_{0 i}\right)+\gamma_{1} \text { Stim }_{i j}+\gamma_{2} \text { Cluster }_{i j}+\varepsilon_{i j}
$$

random effect; Stim stands for Stimulation condition (sham, theta, gamma), Cluster stands for the

omitted for conciseness.

For the analysis of Response Time (RT), we compared covariance structures on

275 Maximum Likelihood (ML) estimations using AIC. For these analyses, the best fit was using a

276 Heterogeneous Toeplitz (TPH) covariance structure. 
structure used for Value and Risk were Heterogeneous Compound Symmetry (CSH). The final model to analyze Probability scores used a TPH covariance structure for repeated measures.

\section{EEG analyses}

We preprocessed the data separately for low (1-20 Hz) and high $(20-90 \mathrm{~Hz})$ frequencies.

284 For low frequencies, a fast Fourier transformation was performed with hanning tapers and output

285 frequencies between 1 and $20 \mathrm{~Hz}$. For high frequencies, a fast Fourier transformation was

286 performed with discrete prolate spheroidal sequences (DPSS) tapers, smoothing factor of $5 \mathrm{~Hz}$ and

287 output frequencies between 20 and $90 \mathrm{~Hz}$. Then data were Log normalized to control for 288 discrepancies driven by individual variability (32).

To look for differences in theta and gamma power before and after the stimulation

290 protocols, the power spectra were averaged for the before and post stimulation measurements.

291 Theta-band was defined between 5 and $8 \mathrm{~Hz}$, with $1.5 \mathrm{~Hz}$ above and $1.5 \mathrm{~Hz}$ below the stimulation

292 frequency $(6.5 \mathrm{~Hz})$, whereas gamma-band was defined between 35 and $90 \mathrm{~Hz}$. Theta and gamma

293 power were analyzed for all channels pre and post stimulation, with focus on the frontal left

294 channels (F1 and F5) around the stimulation focus, frontal right channels (F2 and F6) contralateral

295 to the stimulation.

To investigate whether a change in the hemispheric relationship in theta power took place,

297 we calculated the average of theta power in the right hemisphere minus the average in the left

298 hemisphere, named frontal asymmetry (right - left) (9). Moreover, we compared the changes in

299 theta as well as gamma power in the parietal channels before and after stimulation to analyze how

300 focal were the stimulation effects. 
The effects of stimulation in each condition were compared within participants for the intervals of 1 minute and three minutes. Signal processing and EEG data preprocessing were conducted using Matlab (The Mathworks Inc., Massachusetts, USA)custom scripts and the

304 Fieldtrip toolbox (28). The difference in theta power across conditions was correlated with the

305 behavioral results both using the theta-asymmetry before stimulation as covariate and the changes

306 in theta and gamma frequencies as dependent variables by performing a repeated measures

307 ANCOVA with Bonferroni correction.

308 Results

\section{Behavioral results}

\section{Main results: Risk}

To analyze the effects of stimulation on risk-taking behavior, measured as the average

312 standard deviation of the chosen option (as described above), we ran a Linear Mixed Model

313 analysis using stimulation protocol as factor. The estimated fixed effects showed a significant

314 reduction of -0.301 on risk-taking behavior during theta-band stimulation, $\mathrm{t}(66.693)=-2.035$,

$315 \mathrm{p}=.046, \mathrm{SE}=.148$, compared to sham (Fig 4). Moreover, gamma stimulation did not affect the

316 participant's average risk-taking significantly compared to sham, $\mathrm{t}(69.992)=-1.224, \mathrm{p}=.225, \mathrm{SE}=$

317.100 , confirming that the effects observed are frequency specific. conditions (Theta [6,5 Hz], Gamma [40 Hz] and Sham). Error bars depict SEM. Risk can vary between 11.750 and 36.150 . 


\section{Probability scores} significant main effects for stimulation, $F(2,47.287)=.762, p=.921$. The estimated fixed effects

327 analyses did also not yield significant effects of theta-band stimulation, $\mathrm{t}(35.078)=.316, \mathrm{p}=.754$,

$328 \mathrm{SE}=.014$, or gamma stimulation, $\mathrm{t}(67.703)=-.801, \mathrm{p}=.426, \mathrm{SE}=.009$, when compared to sham,

329 meaning that no significant differences in the probability scores were observed after the different

330 stimulation protocols.

333 Mixed Model Procedure with Value as the dependent variable, which yielded a non-significant

334 main effect of stimulation, $F(2,91.890)=2.427, p=.094$. Further analyses of estimated fixed effects

335 yielded significant effects of Theta stimulation on value, with a reduction of -0.674 compared to 336 sham, $\mathrm{t}(64.331)=-2.130, \mathrm{p}=.037, \mathrm{SE}=.316$. No significant effects were observed after Gamma 337 stimulation $\mathrm{t}(71.190)=-1.270, \mathrm{p}=.208, \mathrm{SE}=.207$, compared to sham. This means that there was a 338 significant reduction in the average value chosen by the participants due to the Theta stimulation, 339 confirming that this is a frequency exclusive effect (Fig 5). These findings reinforce the strong 340 relationship between risk-taking behavior and valuation since both processes were affected by the 341 same pattern of stimulation. 


\section{Response Time}

To analyze the participant's response time (RT), we used a Linear Mixed Model with RT

347 as dependent variable. The results show strong significant effects of stimulation, $F(2,50.240)$

$348=35.803, \mathrm{p}<.001$.

349 Estimated fixed effects analyses yielded significant results for theta stimulation, $\mathrm{t}(24.259)$

$350=5.161, \mathrm{p}<.001, \mathrm{SE}=.066$ and nearly significant effects for gamma stimulation, $\mathrm{t}(63.862)=1.880$,

$351 \mathrm{p}=.065, \mathrm{SE}=.027$, when compared to sham. Theta stimulation led to an increase of $41.11 \%$ in

352 response time (compared to sham). This implies that the theta stimulation led to an increase in the

353 deliberation time, which cannot be attributed to the stimulation per se, since this effect was only

354 marginally significant in the gamma stimulation condition. Details can be observed in Fig 6, where

355 response time is plotted against contrast, or trial difficulty level based on the cluster division

356 previously explained, from easier decisions (which are clear, with big differences in EV between

357 Pink and Blue) to difficult decisions in which the mental calculation to define the most

358 advantageous option is more challenging.

Fig 6 - Average Response Time

Presented in seconds by trial difficulty (easier trials have a higher difference in expected value between the trials) by Stimulation protocol (Error bars depict SEM).

\section{EEG results}

\section{Theta-band entrainment}

To investigate the effects of theta-band stimulation on EEG results, we ran a repeated

366 measures ANOVA with theta power as dependent variable. The repeated Measures ANOVA used

367 a 3 (stimulation condition: theta, gamma and sham) by 2 (time: before and after stimulation) by 6 
(theta power averaged over 3 minutes on each electrode: F1, F5, F2, F6, P5 and P6) within subject design, with Bonferroni correction for multiple comparisons. average of -0.135 to an average of -0.056 after stimulation, $F(1,6)=3.383, p=.009$. There was no significant main effect of stimulation, $\mathrm{F}(1,12)=.824, \mathrm{p}=.627$, and no significant interaction effect between stimulation and time, $\mathrm{F}(1,12)=.822, \mathrm{p}=.629$ (for descriptives please refer to S2 Table). ANOVA using frontal asymmetry as dependent variable. There was no significant effect of stimulation on frontal asymmetry, $\mathrm{F}(1,2)=1.191, \mathrm{p}=.168$; time, $\mathrm{F}(1,1)=.059, \mathrm{p}=.810$ or of the interaction between time and stimulation, $\mathrm{F}(1,2)=.807, \mathrm{p}=.457$. as dependent variable the difference in theta power between the first minute before stimulation and the first minute immediately after it. We used a 3 (stimulation conditions: theta, gamma and sham) by 7 (theta power difference on each electrode: F1, F5, F2, F6, P5, P6 and change in frontal asymmetry) within subject design, with Bonferroni correction for multiple comparisons. These analyses yielded a significant effect of stimulation, $\mathrm{F}(1,12)=4.440, \mathrm{p}<.001$. change (pre-post) when compared to sham $\mathrm{t}(2)=2,528, \mathrm{p}=.012$. However, this effect is mainly 
391 after the task to a positive score when 3 minutes are analyzed. This indicates that during the first

392 minute after the task execution there was a higher theta power in the left hemisphere, which

393 changed during the following minutes, since the analyses including 3 minutes after task indicate a

394 higher theta power in the right hemisphere. More details can be seen in Fig 7. Moreover, the only

395 frequency specific effect observed after theta-band stimulation was during the first minute post

396 stimulation in F6 (contralateral to the stimulation focus) $(\mathrm{t}=3.876, \mathrm{p}<.001)$.

Fig 7 - Frontal theta-band asymmetry difference during 1 and 3 minutes after stimulation

402 theta power in F1, F3, F5, F6, P2 and P6 and the behavioral responses (probabilities chosen, 403 average value chosen, risk and response time). The level of asymmetry before or after the 404 stimulation did not significantly correlate with either of the behavioral measures. Theta power in $405 \mathrm{~F} 1$ and F2 were significantly correlated to the probabilities chosen $(\mathrm{r}=.107, \mathrm{p}=.018$ and $\mathrm{r}=.013$ 406 and $\mathrm{p}=.013$ respectively), although there were no significant effects of stimulation on the 407 probabilities chosen by the participants. The results also indicate trends regarding the correlations 408 between theta-power in F1 and F2 and the average values chosen $(\mathrm{r}=.075, \mathrm{p}=.098$ and $\mathrm{r}=.077$ and $409 \mathrm{p}=.090$ respectively) and between theta-power in these same electrodes and risk $(\mathrm{r}=.080, \mathrm{p}=.077$ 410 and $\mathrm{r}=.082, \mathrm{p}=.071$ respectively).

411 The inclusion of asymmetry of theta-power in any of the electrodes in the regression 412 models used to analyze the behavioral results did not improve the fit of these models and therefore 413 was discarded. 


\section{Gamma-band entrainment}

417 averaged over 3 minutes on each electrode: F1, F5, F2, F6, P5 and P6) within subject repeated 418 measures ANOVA, with Bonferroni correction for multiple comparisons. No significant effect of 419 stimulation condition $\mathrm{F}(1,12)=0.845, \mathrm{p}=.604$, nor of time, $\mathrm{F}(1,6)=1.045, \mathrm{p}=.424$ or the interaction 420 between stimulation and time, $\mathrm{F}(1,12)=1.035, \mathrm{p}=.465$ was observed,

\section{Discussion}

The present study aimed at investigating the functional relationship between frontal theta-

423 band oscillations and risk-taking behavior. Although previous studies $(9,10)$ have shown a 424 correlation between resting state frontal theta band asymmetry and risk-taking behavior, no direct 425 causal relationship has thus far been shown. We hypothesized that theta oscillations underlie the 426 neuronal communication for recruiting DLPFC when the decision-making process includes risk,

427 being fundamental for the modulation of risk-taking behavior (12). We therefore expected theta428 band stimulation to cause a reduction in risk-taking behavior and that this effect is frequency 429 specific.

As predicted, we were able to effectively reduce risk-taking behavior in healthy

431 participants using theta-band tACS over the left DLPFC, compared to sham and gamma-band 432 stimulation. These findings confirm the functional relationship between theta-band frequencies 433 and risk-taking behavior regulation, being a fundamental part of the electrophysiological 434 mechanism responsible for this modulation. Theta band tACS leads to a significant decrease of $4351.12 \%$ of risk taking behavior compared to sham. This was not the case during gamma stimulation. 
436 To our knowledge, our study is the first to show the frequency specificity of this effect. Moreover,

437 we observed a significant reduction on value sensitivity due to theta-band (and not gamma)

438 stimulation, meaning that participants opted for lower values after theta-band stimulation

439 compared to the results obtained in the sham or gamma conditions. These results are in line with

440 previous studies, where participants became more risk-averse after non-invasive brain stimulation

441 with reduced sensitivity to value (22,33-35). However, our study was able to show that also this

442 effect is frequency specific. Therefore, it is expected that theta-frequencies would play a

443 fundamental role in the reduction of value sensitivity, meaning the recruitment of DLPFC as

444 executive control to modulate the VMPFC response to the value (36).

The stimulation did not affect the probabilities chosen by the participants, indicating that

446 the choice of probabilities might be regulated by a different electrophysiological mechanism. Even

447 though our results indicate that probabilities and value are evaluated independently in our brain,

448 behaviorally and in terms of neurological activity these processes are at least strongly correlated

449 (2,29,37). This means that both inputs are considered (bet value and its probabilities) in order to

450 inform the decision process, which justifies the use of standard deviation as an estimation of risk.

451 Our approach considers the option's expected value (meaning the bet's probabilities and value) to

452 estimate risk, which is in our perspective a more naturalistic evaluation of risk. Our findings

453 indicate that participant's reductions on risk-taking behavior were mainly driven by a reduction on

454 the average value sensitivity.

Although we did not have a specific hypothesis regarding the response time, it is 456 interesting to notice that theta stimulation increased response time compared to sham and gamma 457 stimulation. It may be speculated that the increased response time reflects a longer deliberation 458 process (38). 
It is important to note that our results contradict the study by Sela and colleagues

460 (2012).Their results indicated an increase in risk-taking behavior after theta-band stimulation,

461 which might be explained by their choice of the Balloon Analog Task as experimental paradigm.

462 Since this task has a strong factor of impulsivity the effect observed should reflect an increase in

463 impulsivity and not in risk-taking behavior (16). Moreover, they considered the tolerability to

464 losses (measured as sequential explosions) as an indicator of riskier choices (14), which means

465 that their results might also indicate a reduction in loss-aversion. Since our experimental paradigm

466 (MGT) avoids loss-aversion and impulsivity, we may have more directly assessed risk-taking

467 behavior.

In addition to assessing the behavioral effects of our oscillatory brain state

469 neuromodulation on risk taking modulation, we also used EEG to measure oscillatory activity

470 before and after the tACS stimulation. When comparing theta power before and after theta tACS,

471 no significant changes were found, nor did we reveal significant changes in hemispheric theta-

472 band asymmetry after theta-band stimulation. This may seem surprising and in contrast to our

473 behavioral effects being attributed to and interpreted as being caused by tACS-induced increased

474 in left theta power. However, it is important to note here that while behavioral effects were assessed

475 during tACS being applied simultaneously with task execution, the EEG measurements, due to

476 tACS artefacts, were restricted to assessing the oscillatory activity after both the behavioral

477 performance and the tACS stimulation had ended. Especially the latter may be a straightforward

478 explanation for the absence of significant EEG effects in a pre-post tACS design as such effects

479 rely on a significant longer lasting neurophysiological effect of tACS beyond the period of

480 stimulation itself. 
482 from being settled (39). Various previous studies also reported difficulties in establishing longer

483 lasting effects of tACS on excitability or neural plasticity $(13,40-42)$. Considering our results, we

484 may therefore speculate that the EEG effects were only present during the task and stimulation and

485 faded away immediately after tACS had ended. Our post hoc analyses focusing only on the first

486 minute of post EEG measurements after TACS and contrasting these effects to the entire post EEG

487 period indicate time-sensitive changes in theta band asymmetry in line with this speculation. Yet,

488 our study was not designed to conclusively test and other related hypotheses regarding the

489 difference between the immediate versus lasting effects of tACS on neural oscillatory activity.

490 Follow up studies with online measurements using algorithms to remove stimulation artifacts

491 could be used to investigate such possibility, although currently this methodology is still in debate

In addition, we also revealed that the task execution itself had lasting effects on theta band

494 asymmetry as indicated by post hoc analyses of the EEG measures immediately after the task

495 execution in the sham condition. In other words, unrelated to tACS, the mere behavioral

496 performance in risk taking modulation tasks considerably affected theta band asymmetry after task

497 execution had been completed. At the same time, our behavioral results showed no significant

498 correlation with resting-state frontal theta-band asymmetry at baseline, indicating that these effects

499 cannot be explained only by the resting-state frontal asymmetry or by changes in asymmetry due

500 to the stimulation.

The stimulation frequency-specificity of our significant behavioral findings, however,

502 confirming our a priori hypothesis that specifically theta, not gamma or sham, neurostimulation

503 should affect risk taking behavior, clearly represents supporting evidence for the functional 
504 relationship between theta-band stimulation and risk-taking regulation driven by reduction of

505 sensibility to reward. This work also contributes to the understanding of the frontal areas

506 interaction in the regulation of risk-taking behavior, as much as the role of theta-band oscillations

507 in this process. Moreover, it gives insights about the causes of individual differences in risk-taking,

508 granting the analysis of frontal resting state brain activity a potential role to infer differences in

509 individual risk-proneness. This can be used in the construction of more accurate economic models

510 of risk-taking, or contribute to the development of diagnosis and intervention techniques for

511 patients with abnormal risk-taking behavior, since this is characteristic of a range of psychiatric

512 and neurological disorders (43).

\section{Conclusion}

Although it is widely accepted that DLPFC have an important role in risk-taking

516 regulation it is not clear how the recruitment of this area occurs in presence of risk. Theta

517 oscillations are potentially responsible for neuron communication when cognitive control is

518 needed (12). In our study, we provided empirical evidence for the direct functional relationship

519 between prefrontal theta band activity and risk-taking regulation using high definition theta-band

520 tACS with gamma-band entrainment and sham as control. A significant reduction of risk-taking

521 behavior was observed after theta-band, but not gamma-band or sham tACS over left DLPFC,

522 confirming the specific role of theta frequencies in risk-taking behavior regulation. Such findings

523 indicate that prefrontal theta-band oscillations are potentially the basis of communication between

524 frontal areas during risk-taking regulation. 


\section{Acknowledgements}

We would like to thank our research assistant Kira Temme for her help during data

528 collection.

\section{Funding sources}

This research did not receive any specific grant from funding agencies in the public, 


\section{References}

534 1. Trimpop R. The psychology of risk taking behavior. North-Holland; 1994. 386 p.

535 2. Kuhnen CM, Knutson B. The neural basis of financial risk taking. Neuron. 2005 Sep

$536 \quad 1 ; 47(5): 763-70$.

537 3. Brand M, Recknor EC, Grabenhorst F, Bechara A, Brand M, Recknor EC, et al. Decisions

$538 \quad$ under ambiguity and decisions under risk : Correlations with executive functions and

539 comparisons of two different gambling tasks with implicit and explicit rules of two

540 different gambling tasks with implicit and explicit rules. 2007;3395(November 2017).

541 4. Knoch D, Gianotti LRR, Pascual-leone A, Treyer V, Regard M, Hohmann M, et al.

542 Disruption of Right Prefrontal Cortex by Low-Frequency Repetitive Transcranial

543 Magnetic Stimulation Induces. 2006;26(24):6469-72.

544 5. Schonberg T, Fox CR, Poldrack RA. Mind the gap : bridging economic and naturalistic

545 risk-taking with cognitive neuroscience. Trends Cogn Sci [Internet]. 2011;15(1):11-9.

$546 \quad$ Available from: http://dx.doi.org/10.1016/j.tics.2010.10.002

547 6. Studer B, Pedroni A, Rieskamp J. Predicting Risk-Taking Behavior from Prefrontal

548 Resting- State Activity and Personality. Siegel A, editor. PLoS One [Internet]. 2013 Oct 7

$549 \quad$ [cited 2019 Jul 30];8(10):1-8. Available from:

$550 \quad$ https://dx.plos.org/10.1371/journal.pone.0076861

551 7. Floden D, Alexander MP, Kubu CS, Katz D, Stuss DT. Impulsivity and risk-taking

552 behavior in focal frontal lobe lesions. 2008;46:213-23.

553 8. Galvan A, Hare TA, Parra CE, Penn J, Voss H, Glover G, et al. Earlier Development of 554 the Accumbens Relative to Orbitofrontal Cortex Might Underlie Risk-Taking Behavior in 555 Adolescents. 2006;26(25):6885-92. 
556 9. Gianotti LRR, Knoch D, Faber PL, Lehmann D, Roberto D, Diezi C, et al. Tonic Activity

557 Level in the Right Prefrontal Cortex Predicts Individuals ' Risk Taking. 2009;20(1):33-8.

558 10. Studer B, Pedroni A, Rieskamp J. Predicting Risk-Taking Behavior from Prefrontal

559 Resting-State Activity and Personality. Siegel A, editor. PLoS One [Internet]. 2013 Oct 7

560 [cited 2019 Jul 30];8(10):e76861. Available from:

$561 \quad$ https://dx.plos.org/10.1371/journal.pone.0076861

562 11. Gallagher M, McMahan R, Schoenbaum G, O’Doherty JP. Orbitofrontal Cortex and

563 Representation of Incentive Value in Associative Learning. J Neurosci [Internet]. 2009

564 Sep 30 [cited 2019 Jul 31];19(15):6610-4. Available from:

565 https://www.jneurosci.org/content/29/39/12315.short

566 12. Cavanagh JF, Frank MJ. Frontal theta as a mechanism for cognitive control. Trends Cogn

Sci [Internet]. 2014;18(8):414-21. Available from:

http://dx.doi.org/10.1016/j.tics.2014.04.012

569

570

571

572

573

574

575

576

577

578

13. Reato D, Rahman A, Bikson M, Parra LC. Effects of weak transcranial alternating current stimulation on brain activity-a review of known mechanisms from animal studies

[Internet]. Vol. 7, Frontiers in Human Neuroscience. Frontiers Media S. A.; 2013 [cited 2020 Jan 31]. p. 687. Available from:

http://journal.frontiersin.org/article/10.3389/fnhum.2013.00687/abstract

14. Sela T, Kilim A, Lavidor M. Transcranial alternating current stimulation increases risktaking behavior in the Balloon Analog RiskTask. 2012;6(February):1-11.

15. Feurra M, Galli G, Rossi S. Transcranial Alternating Current Stimulation Affects Decision Making. Front Syst Neurosci [Internet]. 2012 May 25 [cited 2019 Apr 8];6:39. Available from: http://journal.frontiersin.org/article/10.3389/fnsys.2012.00039/abstract 
16. Lejuez CW, Read JP, Kahler CW, Richards JB, Ramsey SE, Stuart GL, et al. Evaluation of a Behavioral Measure of Risk Taking: The Balloon Analogue Risk Task ( BART ). $2002 ; 8(2): 75-84$.

582 17. Başar E, Başar-Eroglu C, Karakaş S, Schürmann M. Gamma, alpha, delta, and theta oscillations govern cognitive processes. Int J Psychophysiol [Internet]. 2001 Jan 1 [cited 2019 Jul 25];39(2-3):241-8. Available from: https://www.sciencedirect.com/science/article/pii/S0167876000001458

18. Antal A, Alekseichuk I, Bikson M, Brockmöller J, Brunoni AR, Chen R, et al. Low intensity transcranial electric stimulation: Safety, ethical, legal regulatory and application guidelines. Vol. 128, Clinical Neurophysiology. Elsevier Ireland Ltd; 2017. p. 1774-809.

589 19. Rogers R., Everitt B., Baldacchino A, Blackshaw A., Swainson R, Wynne K, et al. Depleted Normal Volunteers: Evidence for Monoaminergic Mechanisms. Neuropsychopharmacology [Internet]. 1999 Apr 1 [cited 2019 Apr 8];20(4):322-39.

20. Deakin J, Aitken M, Robbins T, Sahakian BJ. Risk taking during decision-making in

597 21. Yazdi K, Rumetshofer T, Gnauer M, Csillag D, Rosenleitner J, Kleiser R. Neurobiological 598 processes during the Cambridge gambling task. Behav Brain Res. 2019 Jan 1;356:295304.

600 22. Boggio PS, Zaghi S, Beatriz A, Fecteau S, Pascual-leone A, Fregni F. Modulation of risktaking in marijuana users by transcranial direct current stimulation ( tDCS ) of the 
dorsolateral prefrontal cortex ( DLPFC ). Drug Alcohol Depend [Internet].

23. Fecteau S, Agosta S, Hone-blanchet A, Fregni F, Boggio P, Ciraulo D, et al. Modulation of smoking and decision-making behaviors with transcranial direct current stimulation in tobacco smokers : A preliminary study. Drug Alcohol Depend [Internet]. 2014;140:78-84. Available from: http://dx.doi.org/10.1016/j.drugalcdep.2014.03.036

24. Valasek A, Fecteau S, Pascual-leone A, Campanha C, Fregni F. Modulation of decisionmaking in a gambling task in older adults with transcranial direct current stimulation. 2010;31(July 2009):593-7.

25. Thielscher A, Antunes A, Saturnino GB. Field modeling for transcranial magnetic stimulation: A useful tool to understand the physiological effects of TMS? In: Proceedings plasticity induced by conventional and high-definition $4 \times 1$ ring tDCS: A neurophysiological study. Brain Stimul. 2013 Jul;6(4):644-8.

27. ten Oever S, de Graaf TA, Bonnemayer C, Ronner J, Sack AT, Riecke L. Stimulus presentation at specific neuronal oscillatory phases experimentally controlled with tACS: Implementation and applications. Front Cell Neurosci [Internet]. 2016 Oct 18 [cited 2019

623 28. Oostenveld R, Fries P, Maris E, Schoffelen J-M. FieldTrip: Open Source Software for Jun 21];10(OCT2016):240. Available from: http://journal.frontiersin.org/article/10.3389/fncel.2016.00240/full Advanced Analysis of MEG, EEG, and Invasive Electrophysiological Data. Comput Intell 
Neurosci [Internet]. 2011 [cited 2019 Jun 21];2011:1-9. Available from:

http://www.hindawi.com/journals/cin/2011/156869/

627 29. Tobler PN, O’Doherty JP, Dolan RJ, Schultz W. Reward value coding distinct from risk

628 attitude-related uncertainty coding in human reward systems. J Neurophysiol. 2007 Feb;97(2):1621-32.

30. Myerson RB. Probability Models for Economic Decisions. Thomson/Brooke/Cole., editor. Technometrics. 2005;48(1):159-159.

632 31. Fecteau S, Pascual-leone A, Zald DH, Liguori P, The H, Boggio PS, et al. Activation of Prefrontal Cortex by Transcranial Direct Current Stimulation Reduces Appetite for Risk during Ambiguous Decision Making. 2007;27(23):6212-8.

635 32. Smulders FTY, ten Oever S, Donkers FCL, Quaedflieg CWEM, van de Ven V. Singletrial log transformation is optimal in frequency analysis of resting EEG alpha. Eur J Neurosci [Internet]. 2018 Oct 1 [cited 2019 Mar 20];48(7):2585-98. Available from: http://doi.wiley.com/10.1111/ejn.13854

33. Gilmore CS, Dickmann PJ, Nelson BG, Lamberty GJ, Lim KO. Transcranial Direct Current Stimulation (tDCS) paired with a decision-making task reduces risk-taking in a clinically impulsive sample. Brain Stimul [Internet]. 2018 Mar 1 [cited 2019 Jun

643 34. Fecteau S, Knoch D, Fregni F, Sultani N, Boggio P, Pascual-leone A. Diminishing RiskTaking Behavior by Modulating Activity in the Prefrontal Cortex : A Direct Current Stimulation Study. 2007;27(46):12500-5. making. Brain Stimul [Internet]. 2012 Apr 1 [cited 2019 Jun 20];5(2):77-83. Available 
from: http://www.ncbi.nlm.nih.gov/pubmed/22537866

36. Hare TA, Malmaud J, Rangel A. Focusing attention on the health aspects of foods changes [cited 2019 Apr 8];31(30):11077-87. Available from:

37. Knutson B, Adams CM, Fong GW, Hommer D, Varner J, Kerich M, et al. Anticipation of Increasing Monetary Reward Selectively Recruits Nucleus Accumbens [Internet]. Vol. 21, The Journal of Neuroscience. 2001 [cited 2019 Oct 9]. Available from: www.jneurosci.org.http://www.jneurosci.org/cgi/content/full/5472

38. Rubinstein A. Response time and decision making: An experimental study [Internet]. Vol. 8, Judgment and Decision Making. 2013 [cited 2019 Oct 9]. Available from: http://arielrubinstein.tau.ac.il/papers/RT2012.pdf.

660 39. Strüber D, Rach S, Neuling T, Herrmann CS. On the possible role of stimulation duration 661 for after-effects of transcranial alternating current stimulation. Front Cell Neurosci [Internet]. 2015 Aug 10 [cited 2020 Feb 14];9(AUGUST):311. Available from: http://journal.frontiersin.org/Article/10.3389/fncel.2015.00311/abstract

664 40. Schutter DJLG. Cutaneous retinal activation and neural entrainment in transcranial alternating current stimulation: A systematic review. Neuroimage. 2016 Oct 15;140:83-8.

666 41. Strüber D, Rach S, Neuling T, Herrmann CS. On the possible role of stimulation duration [Internet]. 2015 Aug 10 [cited 2020 Aug 7];9(AUGUST):311. Available from: http://journal.frontiersin.org/Article/10.3389/fncel.2015.00311/abstract 
Experimental Brain Research. Springer; 2019 [cited 2020 Jul 23]. p. 3071-88. Available from: https://doi.org/10.1007/s00221-019-05666-0

673 43. Rao H, Korczykowski M, Pluta J, Hoang A, Detre JA. Neural correlates of voluntary and 674 involuntary risk taking in the human brain: An fMRI Study of the Balloon Analog Risk Task (BART). Neuroimage. 2008;42(2):902-10. 
$\rightarrow$ Theta-band $(6,5 \mathrm{~Hz})$

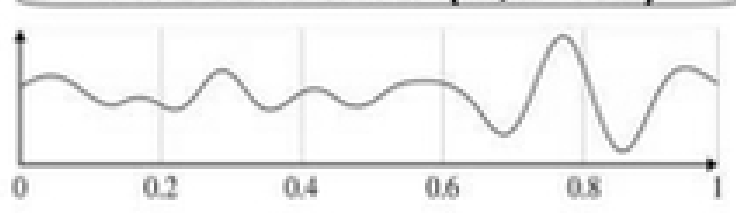

$\rightarrow$ Gamma-band $(40 \mathrm{~Hz})$

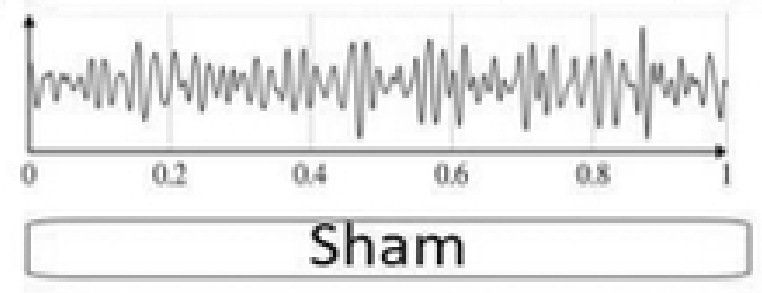

Stimulation

$3 \mathrm{~min}$

$\leftarrow 5$ min

$25-30 \mathrm{~min}$ $3 \mathrm{~min}$

\begin{tabular}{l} 
EEG \\
Pre \\
\hline \\
\hline
\end{tabular}

\begin{tabular}{|c|c|c|c|c|c|c|}
\hline $\begin{array}{c}\text { Task } \\
\text { expla- } \\
\text { nation }\end{array}$ & $\begin{array}{c}10 \\
\text { trai- } \\
\text { ning } \\
\text { trials }\end{array}$ & $\begin{array}{l}50 \\
\text { valid } \\
\text { trials }\end{array}$ & $\begin{array}{l}50 \\
\text { valid } \\
\text { trials }\end{array}$ & $\begin{array}{c}50 \\
\text { valid } \\
\text { trials }\end{array}$ & $\begin{array}{c}50 \\
\text { valid } \\
\text { trials }\end{array}$ & $\begin{array}{l}50 \\
\text { valid } \\
\text { trials }\end{array}$ \\
\hline \multicolumn{7}{|c|}{ Maastricht Gambling Task } \\
\hline
\end{tabular}

EEG

Random

trial selection

Reward

Post selection

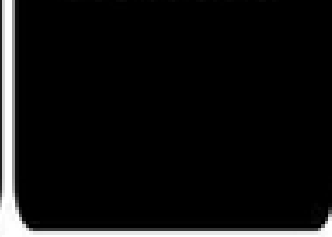



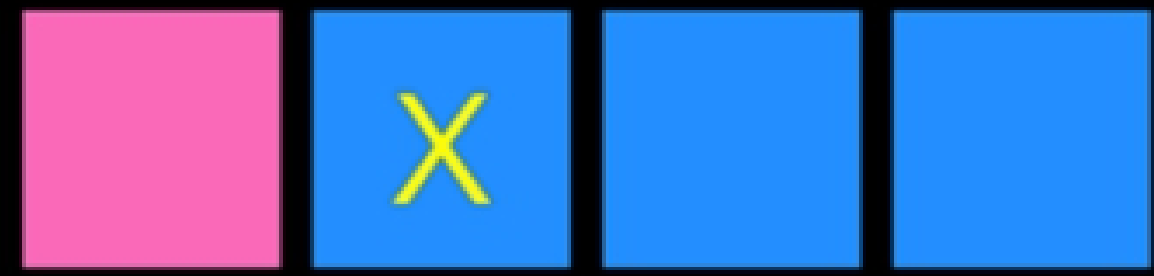

POINTS

50

100

100

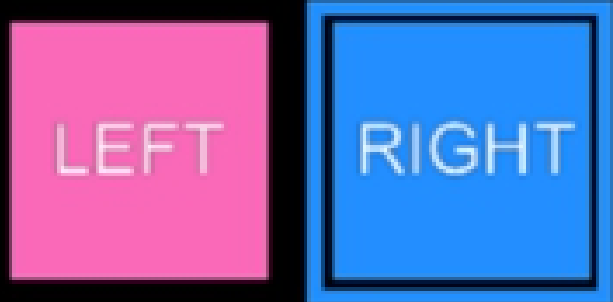

Fig 2 


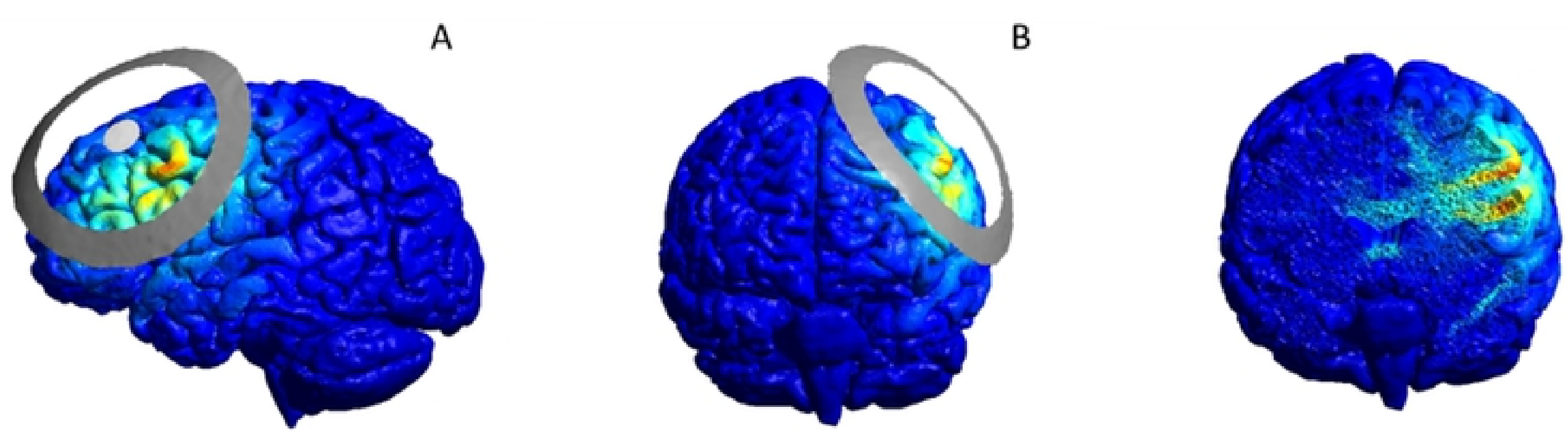

Fig 3 
27,10

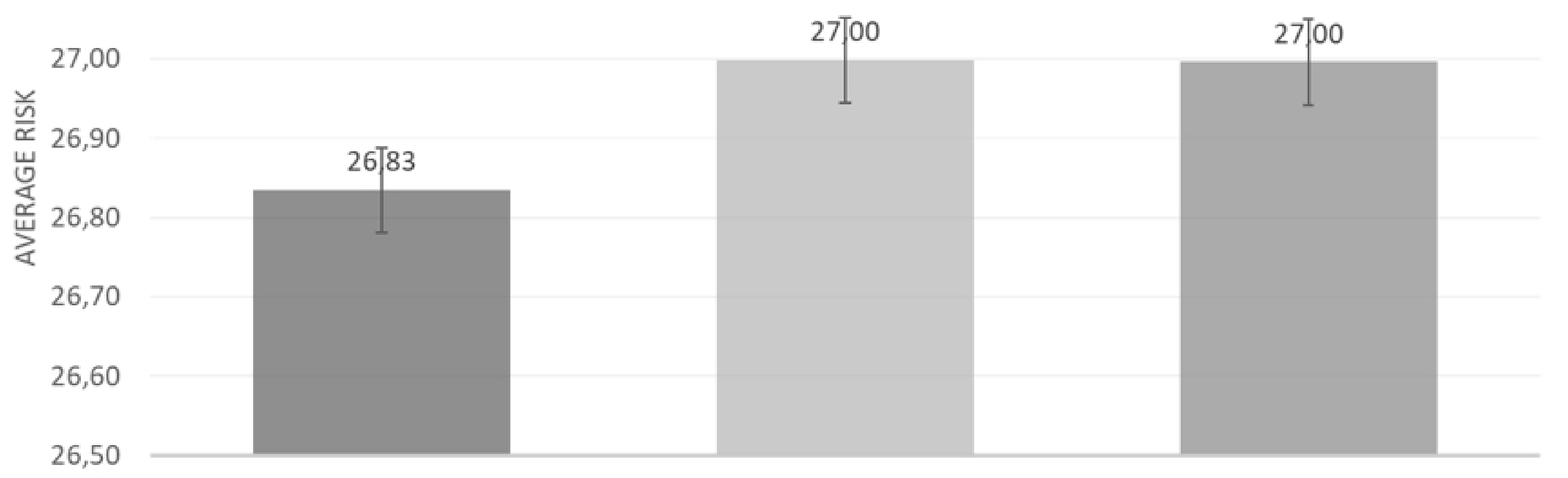

Theta

Gamma

Sham

STIMULATION

Fig 4 
61,00

60,90

60,80

60,70

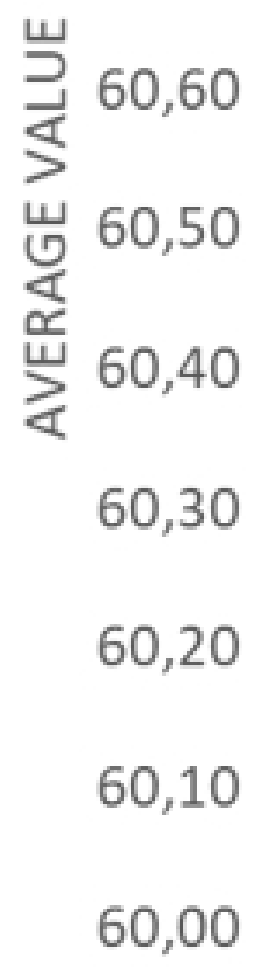

Theta

Gamma

Sham

STIMULATION

Fig 5 


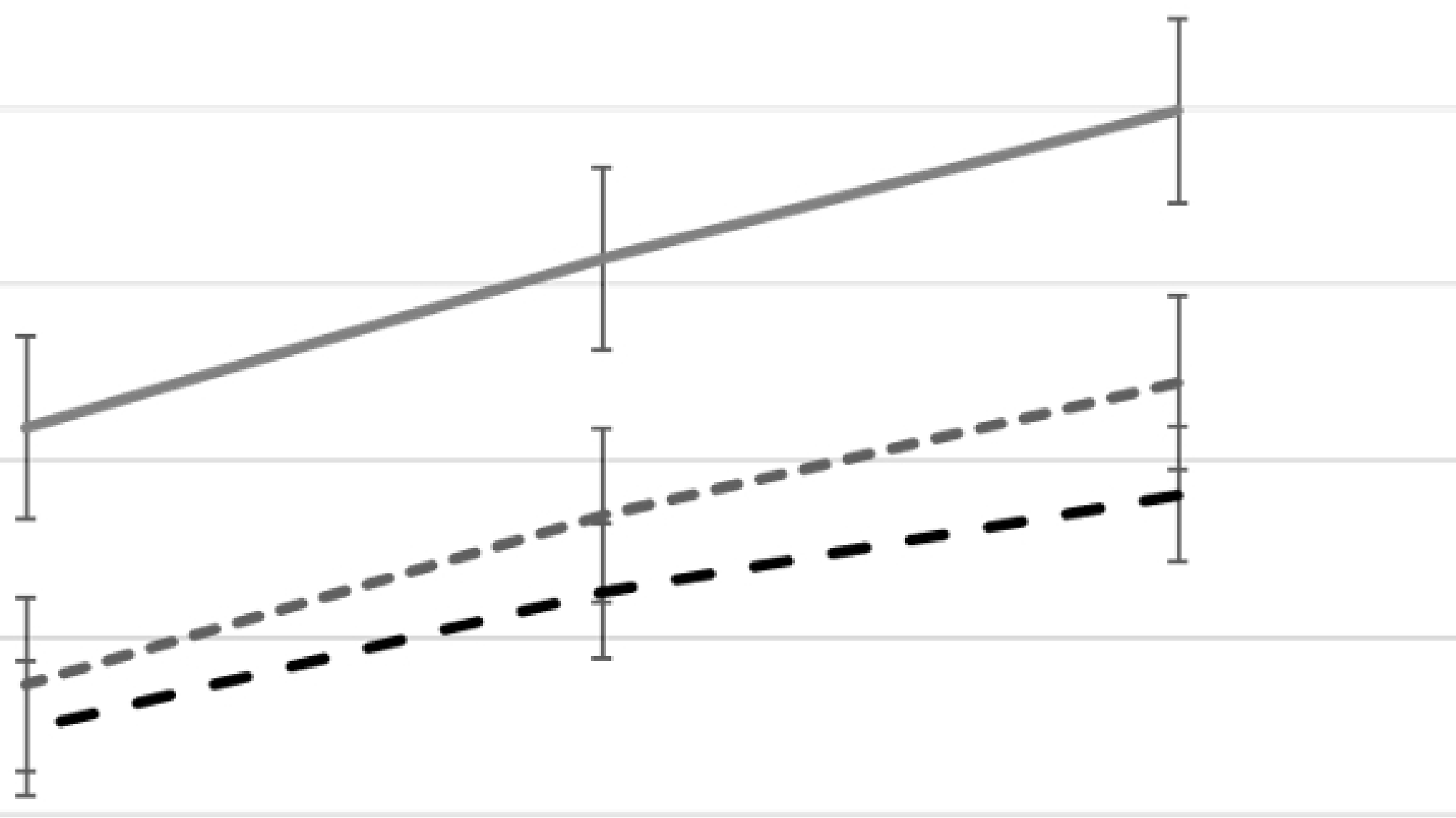

\section{Easy}

Medium

Trial difficulty

Fig 6 


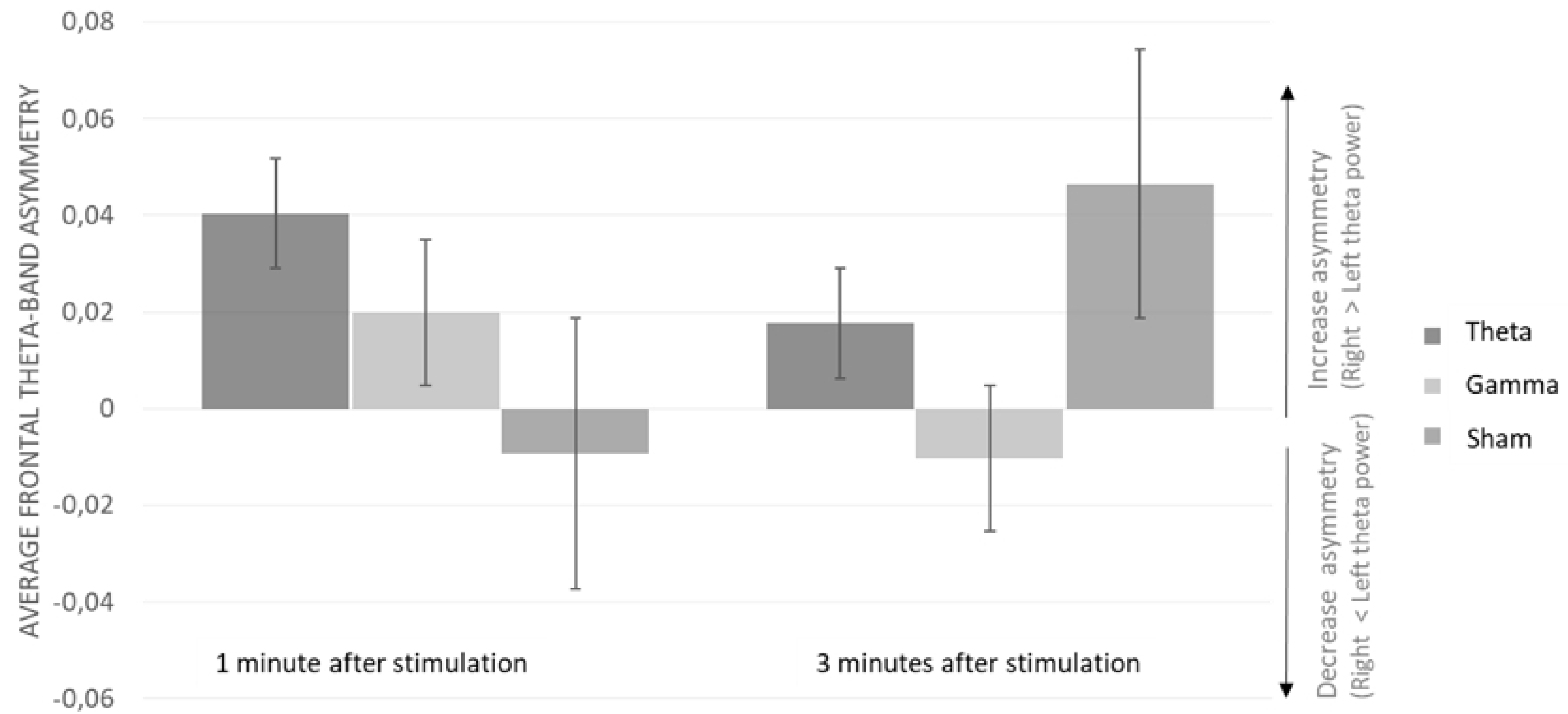
Fig 7 